\title{
Synthesis of Single-Walled Carbon-Nanotubes by Arc-Vaporization under High Gravity Condition
}

\author{
GuoDong Tan, Tetsu Mieno \\ Department of physics, Shizuoka University \\ 836 Ooya, Suruga-ku, Shizuoka 422-8529, Japan
}

\begin{abstract}
Arc-vaporization is one of the common methods to synthesize single-walled carbon nanotubes (SWNTs). However the detailed synthesis mechanism is still not clear, and the synthesis of longer and chirality-selected SWNTs has not been obtained. Here influence of the gravity $G=1 g_{0}, 2 g_{0}$, and $3 g_{0}$ is studied in order to develop the synthesis method of SWNTs. Simultaneously, calculations of heat convection are also carried out by using a fluid simulation program and the results are compared with the experimental results.
\end{abstract}

\section{Keywords:}

Arc discharge, Carbon nanotubes, Diffusion, Heat convection, High gravity, Raman spectrum, Rotating accelerating generator.

\section{Introduction}

It is well known that when single walled nanotubes (SWNTs) are produced by arcvaporization under the normal gravity conditions [1], heat convection flows up carbon clusters in gas phase [2]. This effect will accelerate the upper diffusion of the gas in the reaction chamber, and accelerate the cooling rate of the carbon clusters around the arc 
plasma. In addition, the high temperature region is unsymmetrical by the heat convection. Only upper part of the arc plasma is suitable for synthesis of SWNTs, limiting production region of SWNTs. According to the earlier researches under gravity free condition $\left(G=0 g_{0}\right)[3,4]$, there is no heat convection and the symmetrical and wider high-temperature region can be produced. Around the arc plasma the high temperature region distributes spherically and the volume of high temperature region $(T$ $=2000 \sim 3000 \mathrm{~K})$ is about 14 times larger than that of the normal gravity condition [3]. The production rate of raw carbon soot is about 4 times larger than that of the normal gravity condition $\left(G=1 g_{0}\right)$, and the purity of SWNTs is also better than that of the normal gravity condition [3]. To make clear this gravity effect more precisely, experiment under other gravity conditions is necessary. Here, experiment under high gravity conditions $\left(G=2 g_{0}\right.$ and $\left.G=3 g_{0}\right)$ is carried out. As correct measurement of the hot gas temperature in the reactor and the gas velocity is practically very difficult, fluid calculations of the distribution of the gas temperature and the gas convection velocity around the arc plasma are carried out.

\section{Experimental}

A vacuum chamber made of $4 \mathrm{~mm}$ thick stainless steel, $160 \mathrm{~mm}$ inner diameter and $200 \mathrm{~mm}$ high is used. As shown in Fig. 1, an anode with a cross section of $6 \times 6 \mathrm{~mm}$, made of Ni-Y doped graphite, and a cylindrical graphite cathode $8 \mathrm{~mm}$ in diameter are set in the center of the chamber. A motor driver is set outside of the chamber to control the gap of about $5 \mathrm{~mm}$ between the electrodes.

In order to sample carbon clusters, a particle collector array made of 16 copper cylinders, $1 \mathrm{~mm}$ thick, $8 \mathrm{~mm}$ in inner-diameter and $20 \mathrm{~mm}$ long, is set near the arc 
plasma as shown in Fig. 1. The collection positions are at $z=0,10,20,30,40,50,60$, $70 \mathrm{~mm}$ from the arc center, and at $y=10 \mathrm{~mm}$. 2 CCD cameras are set in front of the chamber to record the images of Mie-scattering [5] from the clusters through a side window.

The anode was sublimated by $\mathrm{DC} 50 \mathrm{~A}$ of an arc discharge in $50 \mathrm{kPa}$ of He gas. After discharge carbon clusters are collected carefully from the each cylinder of the particle collector. And the soot is also sampled at the top cover and the side-wall of the chamber. These carbon clusters are measured by a Raman-spectrometer (JASCO, NR1800 Triple Monochrome Meter Raman. Using $\lambda=532 \mathrm{~nm}$, and $p=70 \mathrm{~mW}$ of $\mathrm{Ar}$ laser ).

The experiment under $G=2 g_{0}$ and $G=3 g_{0}$ is carried out by using a rotating accelerating generator of JAXA (Tsukuba Space Center, Japan. The rotation radius is $6.5 \mathrm{~m}$, the acceleration is $0 \sim 490 \mathrm{~m} / \mathrm{s}^{2}$, rotation frequency $1.6 \sim 83 \mathrm{rpm}$ ) [6-7]. The whole device is set on a head of the rotating accelerating generator as shown in Fig. 2, and the discharge time is 7 minutes for the each gravity condition.

\section{Results and Discussion}

First, the Mie-scattering image is recorded by the CCD camera, where the direct light from the arc plasma is cut by a metal-obstacle plate. It is shown that the heat convection under the high gravity condition becomes stronger, and the upper part of the chamber is filled with "white cloud" made of the clusters after 3 minutes as shown in Fig. 3. It is conjectured that the hot gas is separated into the upper part of chamber, and the colder gas stays in the lower part of the chamber by the strong heat convection. And, a swirl is 
created in the upper part of the chamber. Therefore carbon clusters flown by this swirl stay in the upper part of the chamber to create this "white cloud".

From the images of the arc plasma by the CCD camera, the arc plasma in the high gravity conditions is very stable compared with that of the normal gravity condition. It is conjectured that under the high gravity condition the impurities and sublimated carbon materials are taken off from the electrode surfaces immediately by the stronger heat convection, and the electrode surfaces are always maintained clean.

The weights of carbon soot diffused on the wall and the collectors are measured. The result shows that the soot yield decreases slightly as the gravity is increased. The decrease of the soot yield under the high gravity conditions can be thought to be due to the decrease of the temperature around arc plasma, which should inhibits the sublimation of carbon. Purity and diameters of SWNTs in the soot are measured by the Raman spectrometer. The diameters are measured by radial breathing mode (RBM) of SWNTs, and the purity is estimated by G-band intensity to D-band intensity ratio (G/D ratio). The result shows that 5 sorts of diameters of SWNTs from $1.32 \mathrm{~nm}$ to $1.55 \mathrm{~nm}$ for $G=1 g_{0}$, and 3 sort of diameters from $1.42 \mathrm{~nm}$ to $1.55 \mathrm{~nm}$ for $G=2 g_{0}$ are found at the collector array. But for $G=3 g_{0}$, only one sort of diameter of $1.55 \mathrm{~nm}$ is found at the collector array. For $G=1 g_{0}$ SWNTs are found at $z=10,20,30,50 \mathrm{~mm}$, and the top cover of the chamber (the arc center is set as $z=0 \mathrm{~mm}$ ). For $G=2 g_{0}$ SWNTs are found at $z=20$, and $40 \mathrm{~mm}$, and for $G=3 g_{0}$ at $z=10,30$, and $40 \mathrm{~mm}$. SWNTs of 1.42 $\mathrm{nm}$ diameter are found on the wall of chamber for all of these 3 gravity conditions. These results show that the distribution of diameters decreases and the production region of SWNTs changes as the gravity increases. The SWNTs synthesized by arcvaporization are considered to grow on metallic catalyst particles and the size of the 
metallic catalyst particles would decide the diameter of SWNTs. The calculation results show that by increasing the gravity the cooling rate increases and the high temperature region (where the SWNTs are synthesized) decreases. However in the upper region $(z=$ $10 \sim 40 \mathrm{~mm}$ ), the strong swirl keeps the clusters in the high temperature region for long time, where would be a good gas temperature region for the uniformity of metallic catalytic particles and the production of SWNTs. And the stable production process could decrease the diameter distribution.

Figure 4 shows the Raman spectra and the G/D ratios for the 3 gravity conditions. The G/D ratio is usually used to estimate the purity of SWNTs. Under the $3 g_{0}$ condition highest $\mathrm{G} / \mathrm{D}$ ratio is obtained. While not any large difference of $\mathrm{G} / \mathrm{D}$ ratios is found between $1 g_{0}$ and $2 g_{0}$. Under $G=3 g_{0}$ condition the SWNTs synthesis takes place in a relatively limited region, and the purity is higher than those of $1 g_{0}$ and $2 g_{0}$ conditions. Under the $3 g_{0}$ condition, there is a strong swirl on the upper part of the chamber, and this swirl can keep the clusters in the upper part of the chamber where is good temperature region adequate for the SWNT growth, so that these clusters have longer time to increase the production of SWNTs. It can be assumed that 2 important reactions take place in this swirling region: First, the effective reaction reduces the production of disordered carbon particles and the purity of SWNTs (G/D ratio) will increase. Second, the grown SWNTs will be heated again, and the longer growth time makes more uniform and effective production of SWNTs. Consequently the G/D ratio of the collected sample much increase.

\section{Calculation of the Heat Convection}

Calculations of the heat convections under $G=1 g_{0}, 2 g_{0}$ and $3 g_{0}$ are carried out by using the fluid calculation code (SMAC method [8], Fuji Research Institute Ltd, $\alpha$ - 
Flow) to analyze the distribution of He gas temperature and variation of heat convection velocity around the arc plasma. An axis symmetric cylindrical space according to the experimental condition is divided into 24 pieces and the one piece is divided into $18 \times$ 46 sections, where the non-compression fluid model is solved under the suitable boundary condition. The calculation condition is set as $p_{\mathrm{He}}=40 \mathrm{kPa}$, input electric power $P_{\mathrm{IN}}=1000 \mathrm{~W}$. Figure 5 shows the temperature distributions $(500 \mathrm{~K} \sim 8000 \mathrm{~K})$ of the He gas at $t=0.25 \mathrm{~s}, 1.0 \mathrm{~s}$ and $8.0 \mathrm{~s}$ under $G=1 g_{0}$, and $3 g_{0}$, respectively. Under the $3 g_{0}$ condition, the hot gas above the arc plasma is more localized. And, only the narrow upper part on the arc plasma is kept high temperature condition. Figure 6 shows the He velocity distributions calculated by the SMAC method. There is clear evidence of the gas swirl in the upper chamber under the $3 g_{0}$ condition. This strong swirl would make the "white cloud" in the chamber as shown in Fig. 3.

Figures $7(\mathrm{a}, \mathrm{b}, \mathrm{c})$ show the gas temperature and gas velocity $v_{z}$ calculated for the 3 gravity conditions at $t=8.0 \mathrm{~s}$ and at $y=5.0 \mathrm{~mm}$ when the heat convection is stabilized for the 3 conditions. Figure 7 (d) shows that the cooling rate under $3 g_{0}$ is significantly larger than that of $1 g_{0}$, and is fairly larger than that of $2 g_{0}$ as well. But in all of the 3 cases the cooling rate decreases quickly with $z$. Figures $7(a, b, c)$ show that under the 3 gravity conditions the temperatures of the gas decrease with $z$ in almost same way, but variations of $v_{z}$ under the different gravity conditions show some differences. There, the maximum $v_{z}$ for $1 g_{0}$ is at $z=0 \mathrm{~mm}$, the maximum $v_{z}$ for $2 g_{0}$ is at $z=35 \mathrm{~mm}$, and the maximum $v_{z}$ for $3 g_{0}$ is at $z=45 \mathrm{~mm}$. As the SWNTs with high G/D ratio are found around $z=40 \mathrm{~mm}$ under the $3 g_{0}$ condition, where the temperature settles around 3500 $\mathrm{K}$, it is conjectured that both the gas temperature and the cooling rate are important for 
synthesis of SWNTs. And the swirling would affect the growth of SWNTs by moving the hot carbon clusters in the synthesizing region (high temperature region ) again.

\section{Summary}

The influence of gravity for synthesis of single walled nanotubes by the arc vaporization is studied.

1) The production rate of the carbon soot decreases slightly as the gravity increases.

2) Under the high gravity conditions, the high temperature gas $(\mathrm{He})$ is flown to the upper part of the chamber above the arc plasma by the strong heat convection.

3) Under the high gravity condition $\left(G=3 g_{0}\right)$, SWNTs are produced with relatively higher purity in the relatively limited region, and significant differences in quality and yield of SWNTs are not found between $G=1 \mathrm{~g}_{0}$ and $G=2 g_{0}$.

4) The calculations show that the cooling rate and velocity of He gas are influenced significantly by the gravity. Under $G=3 g_{0}$ the largest cooling rate is obtained, and the peak of gas velocity shifts to upper position. And this shift of peak of gas velocity would correspond to the shift of synthesis region of SWNTs. However, the strong gravity makes the swirl on the arc plasma, which is observed as the "white cloud", and it would increase the production rate of SWNTs in the limited region.

\section{Acknowledgements}

We would like to thank Japanese Aerospace Exploration Agency (JAXA) and AES Co. for operation of the rotating acceleration generator. 


\section{References}

[1] H. Dai. Carbon Nanotubes (eds. M.S.Dresselhaus, G. Dresselhaus, Ph. Avouris). (Springer-Verleg, New York. 2000) P. 30.

[2] T. Mieno, Plasma Phys. Control. Fusion 46 (2004) 211.

[3] T. Mieno, New Diamond Frontier Carbon Technol, 16 (2006) 139.

[4] T. Mieno and M. Takeguchi, J. Appl. Phys, 99 (2006) 104301.

[5] K. koga, S. Iwashita and M. Shiratoni, J. Plys. D. Appl. Phys, 40 (2007) 2267.

[6] G. Tan and T. Mieno, Proc. Int. Conf. Asia MRS (IUMRS-ICA 2008) (December 9-15, 2008) RP-10, Nagoya, Japan.

[7] G. Tan and T. Mieno, Proc. 2nd Int. Conf. on Plasma-Nano Technol. \& Sci. (ICPLANTS 2009) (January 22 23. 2009), P-10, Nagoya, Japan.

[8] R. Peyret and T.D. Taylor, Computational Methods for Fluid Flow, (Springer. New York, 1983) p.160. 


\section{Figure captions}

Fig. 1 Schematic of the vacuum chamber (front view). A particle collector array (right side) composed of 16 copper cylinders, $1 \mathrm{~mm}$ thick, $8 \mathrm{~mm}$ in diameter, $20 \mathrm{~mm}$ long is set at $x=0 \mathrm{~mm}, y=10 \mathrm{~mm}$. And the center of the bottom cylinders are set at $z=0 \mathrm{~mm}$.

Fig. 2 The vacuum chamber is suspended on a head of a rotating accelerating generator, on which a motor driver and 2 video cameras are set.

Fig. 3 A typical image of the Mie-scattering by the diffusing clusters (side view) for $G$ $=3 g_{0}$ at $t=3 \mathrm{~min}$. The particle collector array is shown in the back side.

Fig. 4 (a) Vertical distributions of G/D ratios for the 3 gravity conditions. Raman spectra at several vertical positions. (b) under $G=1 g_{0}$, (c) under $2 g_{0}$, and (d) under $3 g_{0} . p_{\mathrm{He}}=50 \mathrm{kPa}, I_{d}=40 \mathrm{~A}$ and $t_{d}=20 \mathrm{sec}$.

Fig. 5 Temperature distributions of He gas by calculation (side view). (a) $G=1 g_{0}, t=$ $0.25 \mathrm{~s}$, (b) $G=1 g_{0}, t=1.0 \mathrm{~s}$, (c) $G=1 g_{0}, t=8.0 \mathrm{~s}$, (d) $G=3 g_{0}, t=0.25 \mathrm{~s}$, (e) $G$ $=3 g_{0}, t=1.0 \mathrm{~s}$, (f) $G=3 g_{0}, t=8.0 \mathrm{~s} . p_{\mathrm{He}}=40 \mathrm{kPa}$ and $P_{\mathrm{IN}}=1000 \mathrm{~W}$.

Fig. 6 Velocity distributions of He gas by calculation (side view). (a) $G=1 g_{0}, t=0.25 \mathrm{~s}$, (b) $G=1 g_{0}, t=8.0 \mathrm{~s}$, (c) $G=3 g_{0}, t=0.25 \mathrm{~s}$, (d) $G=3 g_{0}, t=8.0 \mathrm{~s} . p_{\mathrm{He}}=40 \mathrm{kPa}$ and $P_{\mathrm{IN}}=1000 \mathrm{~W}$.

Fig. 7 Calculations of cooling processes and cooling rates for the 3 gravity conditions. $(\mathrm{a} \sim \mathrm{c})$ the variations of gas temperature and gas velocity at $z=0 \mathrm{~mm} \sim 50 \mathrm{~mm}, t$ $=8.0 \mathrm{~s}$ for the 3 gravity conditions. (d) comparison of the cooling rates for the 3 gravity conditions. $t=8.0 \mathrm{~s}, p_{\mathrm{He}}=40 \mathrm{kPa}$ and $P_{\mathrm{IN}}=1000 \mathrm{~W}$. 


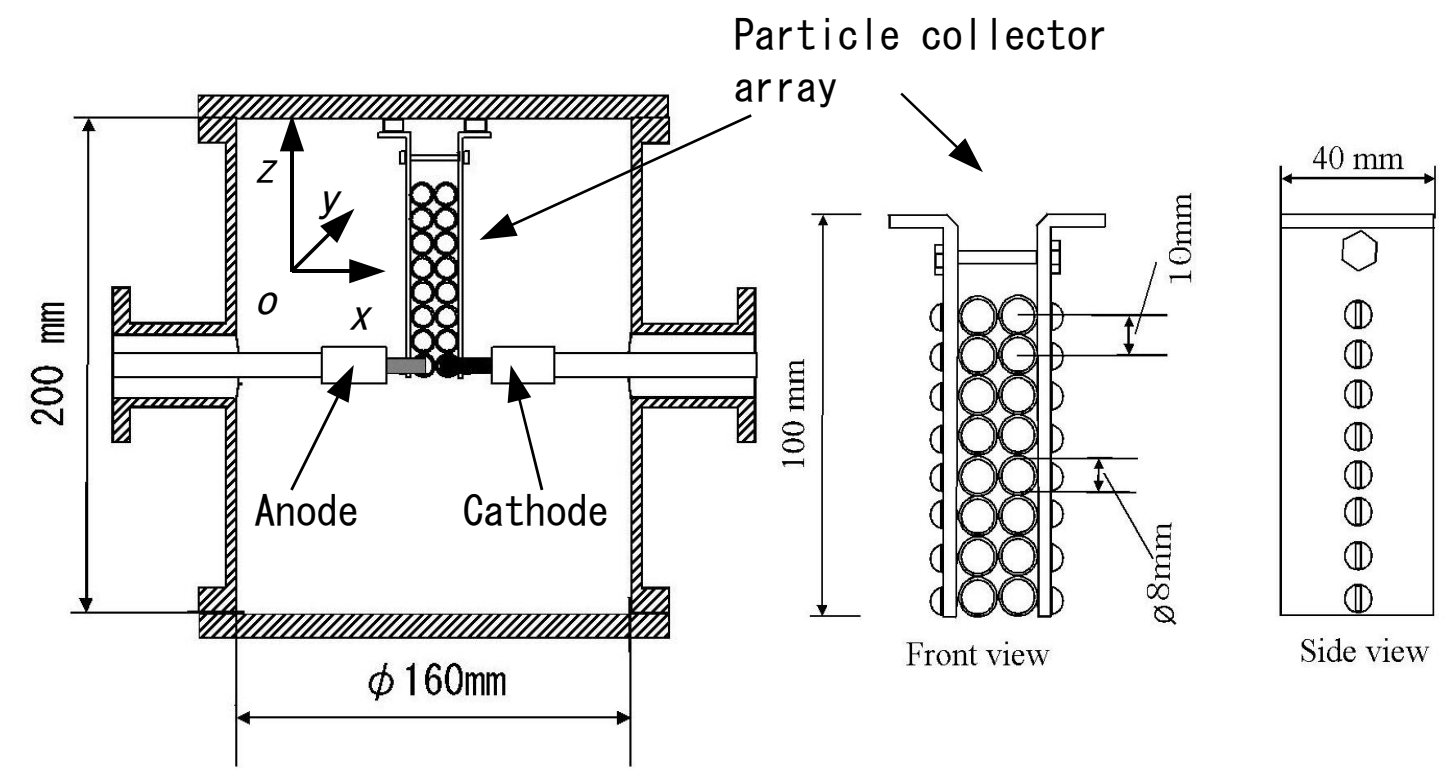

Fig.1 


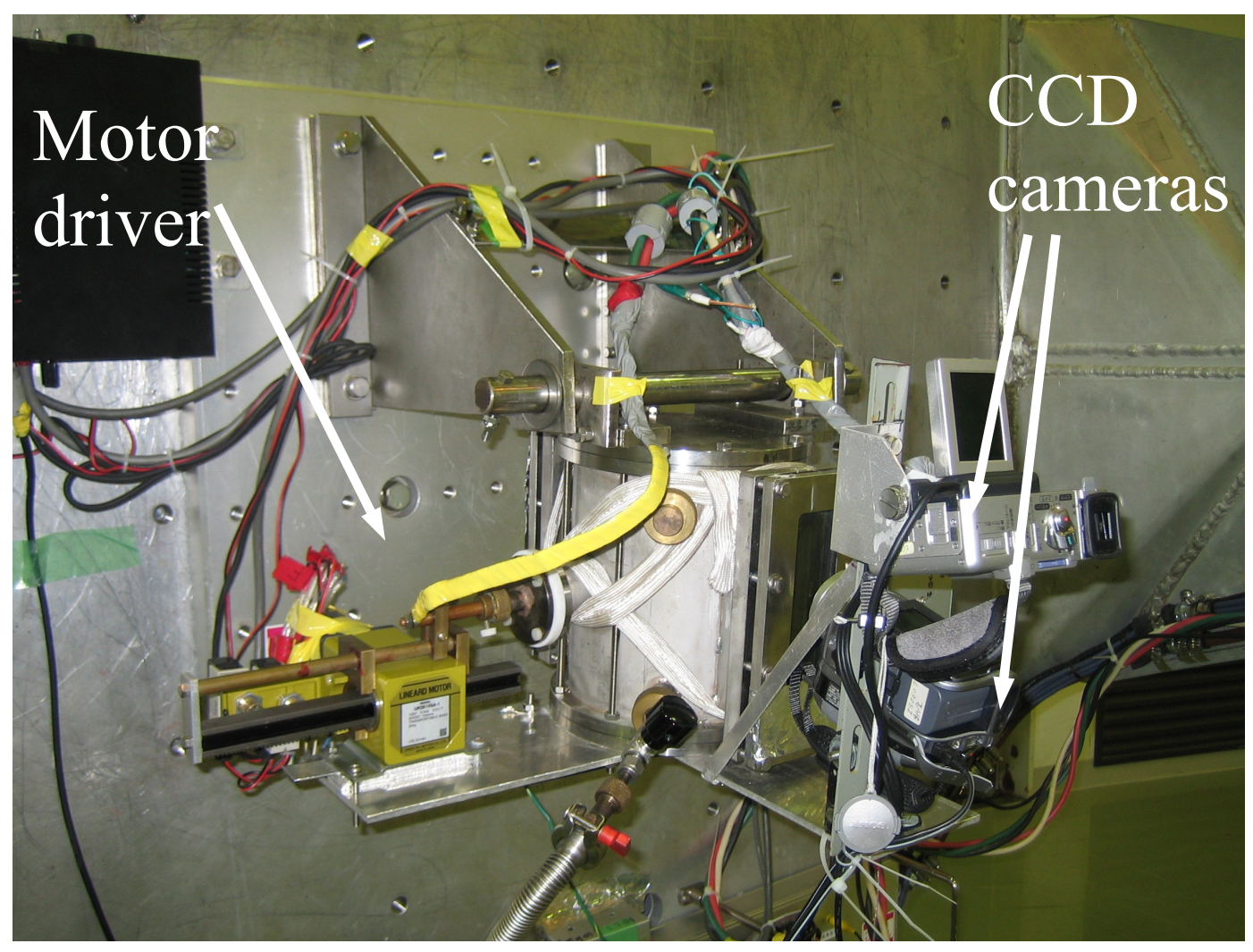

Fig. 2 


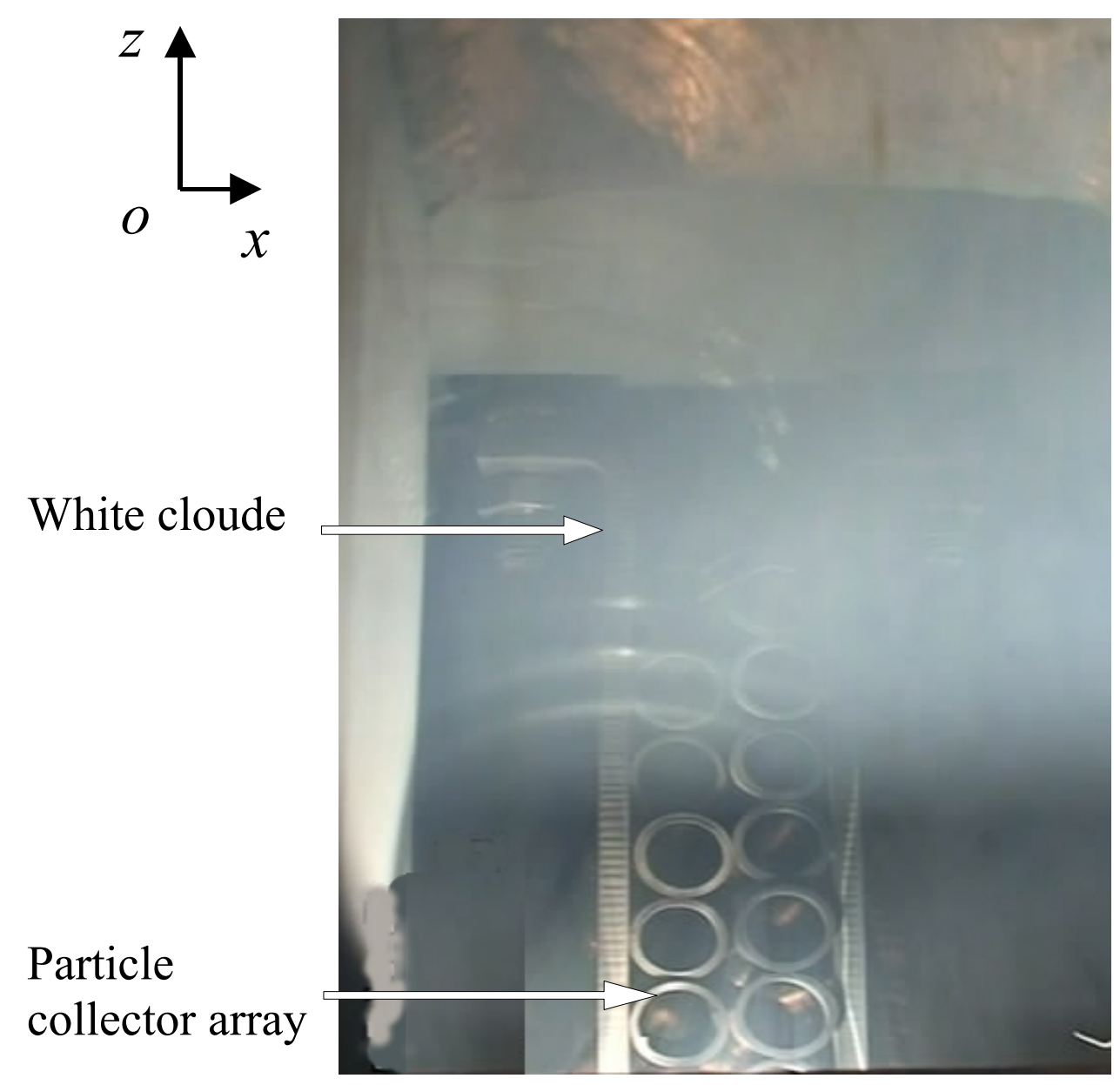

Fig. 3 


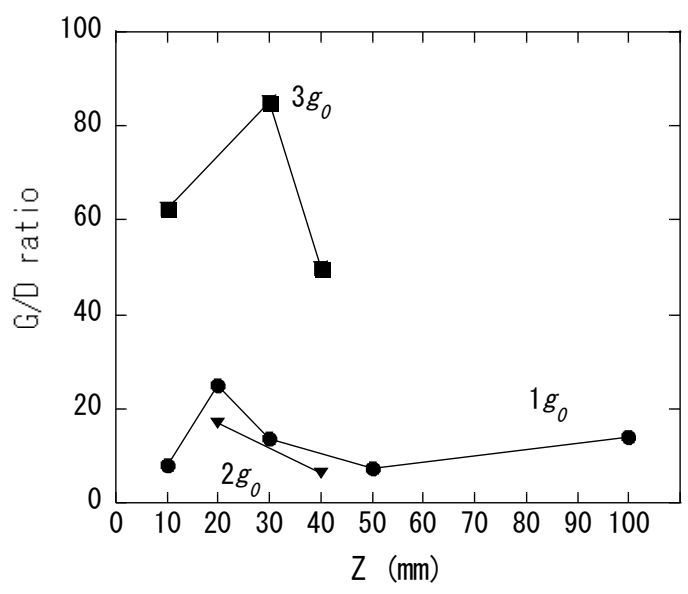

(a)

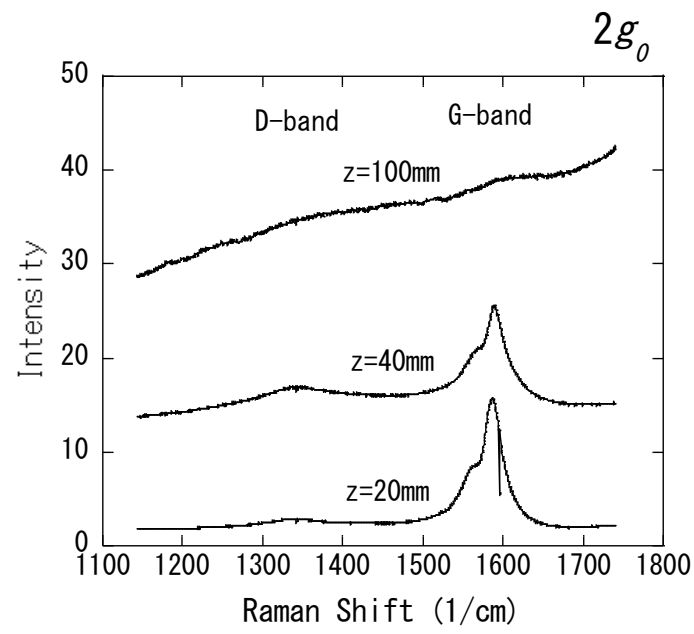

(c)

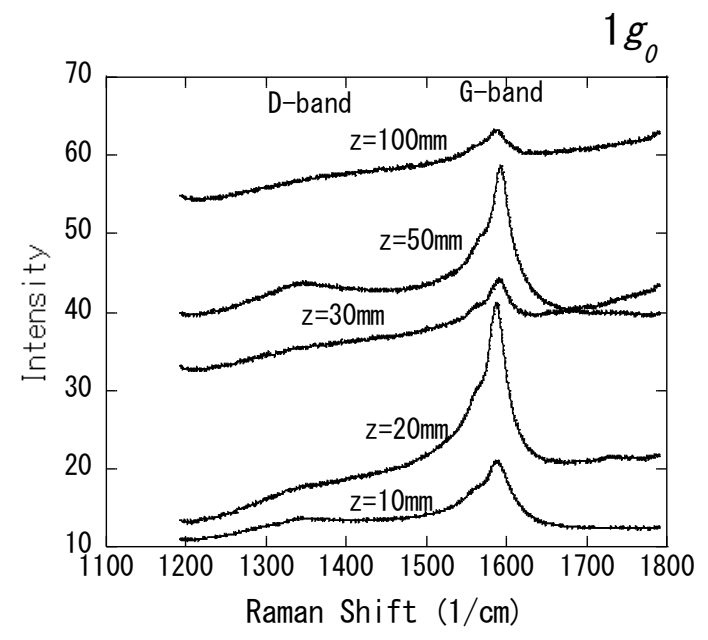

(b)

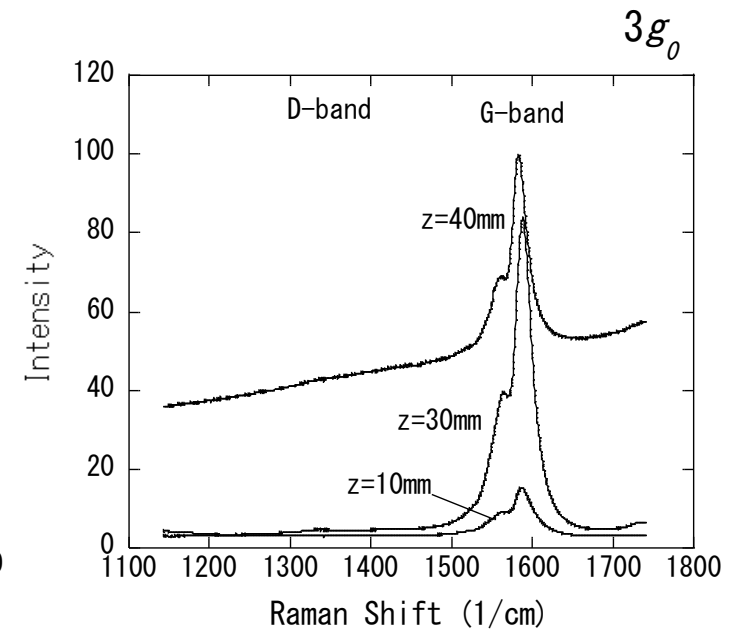

(d)

Fig.4 


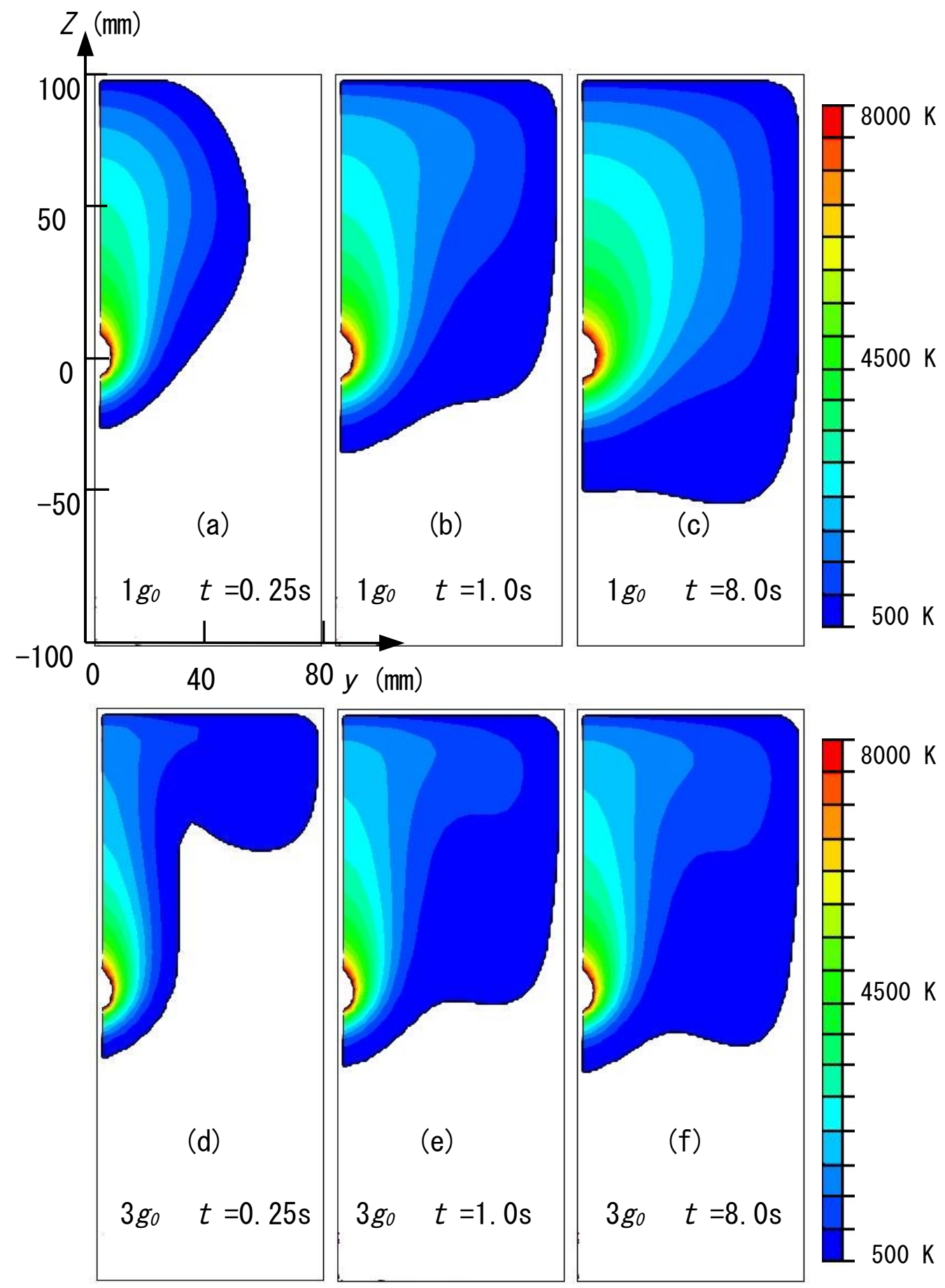

Fig. 5 


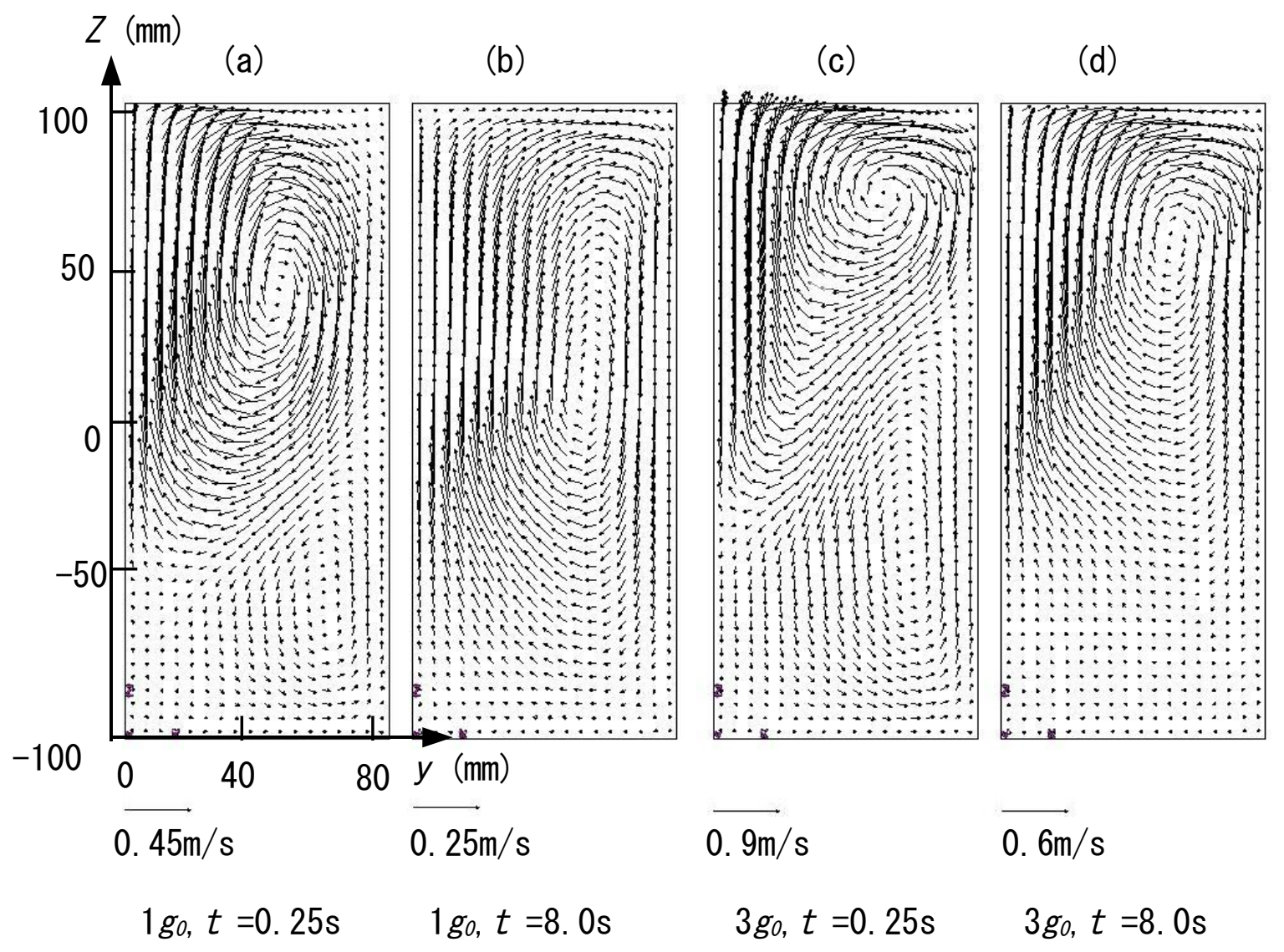

Fig. 6 


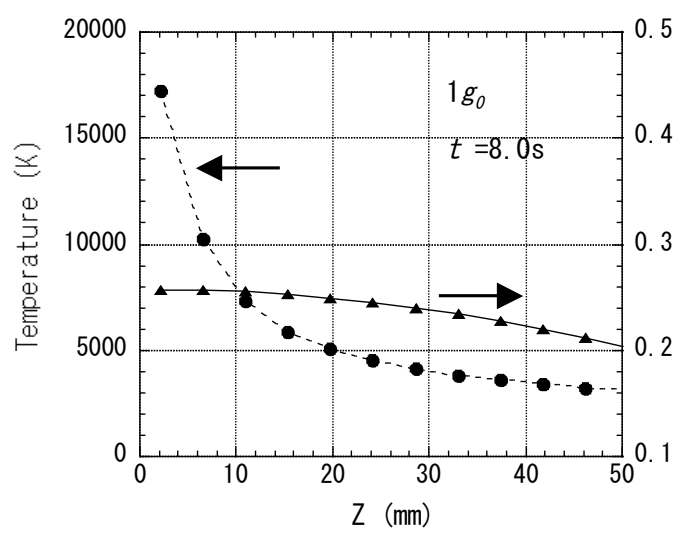

(a)

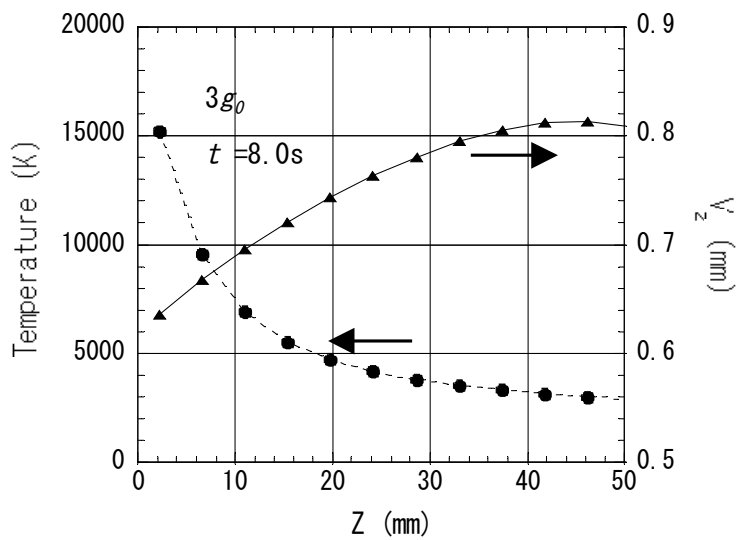

(c)

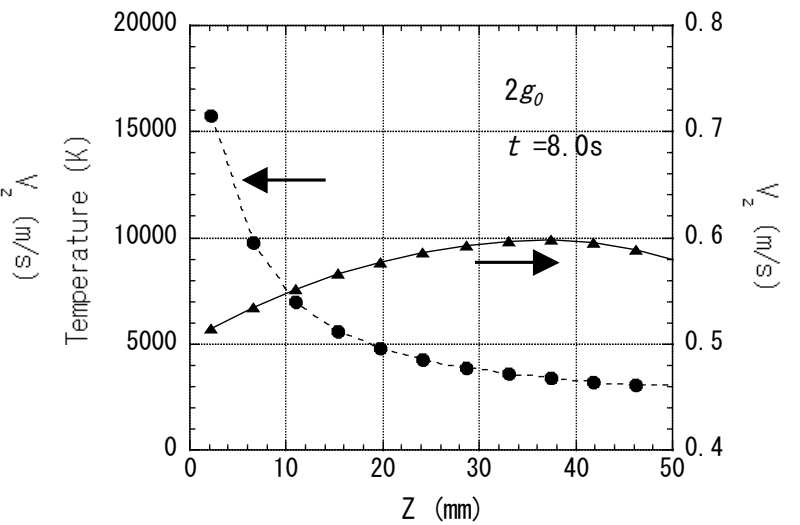

(b)

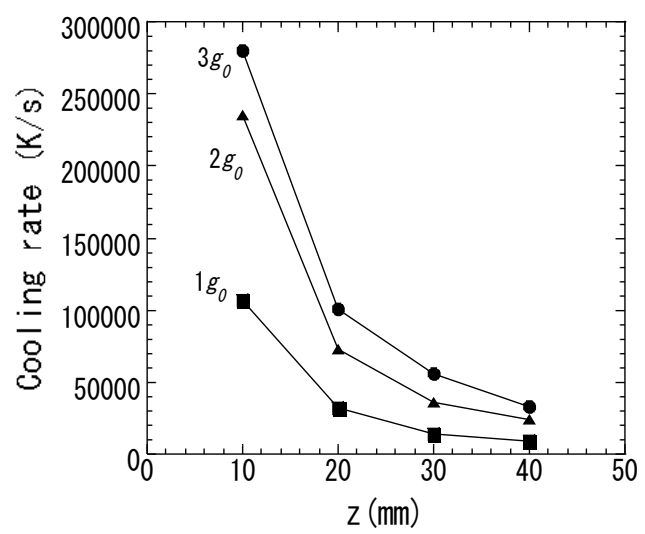

(d)

Fig. 7 
